\title{
ESTUDO DE CASO SOBRE OS ASPECTOS PSICOLÓGICOS APÓS DIAGNÓSTICO DE SARCOMA E REALIZAÇÃO DE AMPUTAÇÃO
}

\author{
A CASE STUDY ABOUT THE PSYCHOLOGICAL ASPECTS AFTER SARCOMA DIAGNOSIS AND \\ AMPUTATION PROCEDURE
}

ESTUDIO DE CASO SOBRE LOS ASPECTOS PSICOLÓGICOS TRAS EL DIAGNÓSTICO DE SARCOMA Y REALIZACIÓN DE AMPUTACIÓN

\section{Deborah Nóbrega da Silva Leal* Aline Mariana Rodicz**}

\begin{abstract}
RESUMO
Os sarcomas sinoviais são tumores raros, marcados por sua agressividade e mau prognóstico. A principal forma de tratamento, além da quimioterapia e radioterapia, ainda é a ressecção cirúrgica do tumor, sendo a cirurgia de amputação uma dessas alternativas. Esse procedimento pode acarretar um grande impacto físico e emocional para o sujeito, não somente pela perda do membro e de sua função, mas também pelos demais aspectos subjetivos que envolvem o próprio procedimento cirúrgico. Por isso, faz-se importante a intervenção da Psicologia ao paciente no pré, trans e pós-cirúrgico, com o objetivo de minimizar suas ansiedades e angústias, possibilitando a expressão dos sentimentos e auxiliando na compreensão da situação vivenciada. Dessa forma, este estudo de caso vem ilustrar a intervenção psicológica a uma paciente durante todo seu processo de adoecimento (diagnóstico, tratamentos e cuidados paliativos) que, por sua vez, proporcionou redução no sofrimento emocional e melhora na qualidade de vida.
\end{abstract}

Palavras-chave: Sarcoma. Amputação. Intervenção psicológica.

\begin{abstract}
Synovial sarcomas are rare tumors, marked by their aggressiveness and bad prognosis. The main treatment is not only chemotherapy and radiotherapy, but also the surgical resection of the tumor, where amputation is one of the alternatives. This procedure can result in great physical and emotional impact in the patient, not only due to the loss of the limb and its function, but also because of other subjective aspects related to the surgical procedure
\end{abstract}

Texto recebido em 15 de janeiro de 2016 e aprovado para publicação em 29 de junho de 2016.

"Mestra em Psico-oncologia pela Universidad Complutense de Madrid, especialista em Cancerologia após Residência no Hospital Erasto Gaertner, graduada em Psicologia.E-mail: obrega.leal@gmail.com.

"Especialista em Psico-oncologia pelo Hospital Erasto Gaertner, graduada em Psicologia pela Universidade do Contestado, Campus Mafra. E-mail: aline_mfa@yahoo.com.br. 
itself. Therefore, it is important to provide psychological care for the patient before, during and after the surgical intervention, aiming at minimizing the patient's anxieties and anguishes, enabling the expression of feelings and helping in the understanding of the situation being experienced. This case study illustrates the need for psychological care for the patient throughout the illness (diagnosis, treatment and palliative care), which, in turn, provides a reduction in the emotional suffering and improvement of life quality.

Keywords: Sarcoma. Amputation. Psychological care.

\section{RESUMEN}

Los sarcomas sinoviales son tumores raros, marcados por su agresividad y mal pronóstico. La principal forma de tratamiento, además de la quimioterapia y radioterapia, sigue siendo la resección quirúrgica del tumor primario, y la cirugía de amputación es una de estas alternativas. La realización de este procedimiento quirúrgico puede resultar en un gran impacto físico y emocional para el sujeto, no solo por la pérdida del miembro y su función, sino también por otros aspectos subjetivos que implican la intervención quirúrgica en sí. Por eso la intervención psicológica es un papel importante para el paciente en los momentos pre-, trans-y postoperatorio, con el objetivo de minimizar sus ansiedades y angustias, posibilitando la expresión de los sentimientos y auxiliando en la comprensión de la situación vivenciada. Por lo tanto, este estudio de caso viene ilustrar la intervención psicológica en un paciente a lo largo de su proceso de enfermedad (diagnóstico, tratamientos y cuidados paliativos) que a su vez, ha proporcionado una reducción de la angustia emocional y una mejora en su calidad de vida.

Palabras clave: Sarcoma. Amputación. Intervención psicológica.

\section{INTRODUÇÃO}

$\mathrm{O}$ câncer pode ser entendido como um conjunto de mais de cem doenças que têm como característica comum o crescimento desordenado de células que invadem os tecidos e órgãos. Essas células são capazes de se espalhar para outras regiōes do corpo, provocando o que é denominado de metástase (Instituto Nacional do Câncer, 2016).

Os tipos de câncer são classificados de acordo com sua localização, sendo que a doença pode surgir em várias partes do corpo. Entre essa tipologia, há os sarcomas de partes moles, que são tumores sólidos que surgem, principalmente, nas extremidades do corpo, assim como, seguidas em ordem de frequência: na cavidade abdominal, retroperitônio, parede do tronco e cabeça e pescoço. Esses tumores compõem um grupo heterogêneo de neoplasias malignas, que, apesar 
de apresentarem um pico de incidência na infância, são mais comuns na idade adulta, representando cerca de $1 \%$ dos cânceres nesse grupo etário, especialmente em maiores de 50 anos (Manoel et al., 2008).

Contudo, assim como citado a respeito dos cânceres em geral, a manifestação de sarcomas de partes moles também está sujeita a classificação tipológica, pela qual se identifica, entre outros, o sarcoma sinovial. Esse tipo de sarcoma é marcado por sua agressividade e mau prognóstico. É um tipo de tumor raro, que apresenta a incidência de aproximadamente $5 \%$ a $10 \%$ dos sarcomas de partes moles e pode aparecer em uma grande variedade de articulações, sendo mais recorrente nos joelhos, nos tornozelos e nos músculos, com predomínio de 75\% nas extremidades inferiores (Corrêa et al., 2005).

Os sarcomas em geral apresentam probabilidade de recidiva local entre 12\% e 31\%, e metástases à distância de 39\% a 54\%, sendo os pulmóes o lugar mais comum. Os principais fatores prognósticos relacionados aos sarcomas de partes moles são o grau de diferenciação histológica do tumor primário, o seu tamanho e a presença ou ausência de metástase (Pellizzon, Salvajoli, \& Novaes, 2002). Na doença metastática, o tratamento geralmente é paliativo e visa ao aumento da sobrevida e à melhora da qualidade de vida.

Os recursos terapêuticos utilizados para o tratamento do sarcoma sinovial são semelhantes aos utilizados para tumores de partes moles de modo geral, sendo a ressecção cirúrgica do tumor primário o principal. No passado, a realização da cirurgia de amputação do membro acometido pelo sarcoma era a regra, porém, nos últimos 20 anos, devido ao tratamento multimodal com uso de radioterapia e quimioterapia, o tratamento conservador com preservação do membro tem sido uma importante alternativa terapêutica. $\mathrm{O}$ objetivo do tratamento conservador com quimioterapia e radioterapia é a ressecção completa do tumor, de forma a manter uma boa função orgânica do membro acometido. Entretanto, apesar das novas alternativas, pode-se afirmar que o tratamento dos sarcomas ainda é um desafio (Pellizzon et al., 2002; Santos, Rezende, Carvalho, \& Nunes, 2015), pois, apesar da utilização de terapia multimodal para tentar reduzir o tumor, nem sempre é possível a preservação do membro (Corrêa et al., 2005). Assim, a amputação cirúrgica ainda é um tratamento muito utilizado, sendo aplicado como último recurso para evitar consequências mais drásticas, tais como a morte do paciente (Quadros, 2010).

Como todo procedimento cirúrgico, a amputação pode despertar sentimentos como o medo e a insegurança, uma vez que elementos como a própria intervenção cirúrgica, a anestesia, o internamento e a dor podem representar uma ameaça para o paciente (Remor, Ulla, \& Arranz, 2003). Além disso, a amputação pode 
ser considerada como uma experiência de perda, na qual o sujeito vivencia profundas transformações físicas e emocionais, podendo ter caráter marcante e mobilizador para a busca de novos significados, de adaptaçóes e de novos referenciais orientadores para a vida do indivíduo (Gabarra \& Crepaldi, 2009).

Aliado ao medo e à insegurança dos enfermos, ocorre ainda o processo de luto que acompanha uma perda, assinala a dor e o sofrimento diante de uma situação nova, indesejável e temida. Além disso, alguns pacientes também podem vivenciar o luto antecipatório diante da descoberta da perda do membro. Esse processo se caracteriza pela apropriação de um conjunto de respostas emocionais específicas semelhantes às vivenciadas no luto, podendo inclusive gerar um maior impacto do que quando a perda é concretizada (Schuler, Zaider, \& Kissane, 2012).

Desse modo, podemos compreender que as reaçóes emocionais, advindas do processo de amputação, são as mais variadas. Para Coutinho e Moura (2004), o paciente pode passar por quatro fases durante esse processo: impacto (desesperança e, ou, negação, na qual a realidade da perda não é vivenciada de fato); isolamento (medo, culpa, raiva, momento em que a amputação se torna aparente e o paciente tende a se isolar); reconhecimento (pode iniciar o processo de autocuidado e agressividade em relação aos cuidadores como reflexo da situação vivenciada); reconstrução (aceitação maior da diferença, ausência e deficiência, surgindo o interesse pela reabilitação).

Contudo, apesar de serem delineados teoricamente, esses estágios não ocorrem de forma predeterminada, linear ou seguindo exatamente o mesmo padrão ou ordem. As diferenças individuais (tais como a idade, a rede social, a estrutura e a dinâmica da personalidade, as causas da amputação e os aspectos sociais e econômicos, entre outros) influenciarão e serão marcantes para a forma como as reações emocionais e o enfrentamento em relação à cirurgia vão se apresentar.

Para além do já citado, é possível ainda perceber um fenômeno alegado por muitos pacientes que realizam a amputação, que é a sensação da presença do membro fantasma e, muitas vezes associada a ela, a dor no membro fantasma, que pode apresentar uma frequência que varia de $60 \%$ a $80 \%$ e acarreta um grande impacto físico e psicológico para o paciente (Flor, Birbaumer, \& Sherman, 2001). Tal sensação do membro fantasma pode ser definida como qualquer sensação de presença do membro que foi amputado, que, algumas vezes, pode se comportar de forma similar ao membro antes da amputação (Faria \& Silva, 2014).

Ademais, também se compreende o fenômeno do membro fantasma pela perspectiva da imagem corporal que é construída de acordo com as ideias, as emoções e as percepções sobre o corpo e suas experiências. Essa imagem corporal pode ser modificada pelo impacto psicológico sofrido por um indivíduo após 
uma amputação, podendo necessitar de adaptações aos novos fatores físicos e sociais. Dessa forma, o membro fantasma pode ser entendido como a "interação entre o que se detecta a nível periférico (corpo) e o que se integra ao nível central (mente), sendo criada então, a aparência final do corpo no sistema nervoso" (Pires \& Bastos, 2013). Assim, podemos considerar que o "fantasma" seria a expressão de uma dificuldade de adaptação a uma ausência súbita de uma parte importante do corpo, visto a percepção usual de ter um corpo completo.

A dor fantasma pode se apresentar de diversas maneiras, como aperto, ardor, compressão ou dor intensa e frequente (Demidoff, Pacheco, \& Sholl-Franco, 2007). Embora sua fisiopatologia ainda não esteja completamente estabelecida, estudos clínicos e experimentais vêm contribuindo, de forma significativa, para o entendimento da dor fantasma, havendo teorias centrais, espinhais e periféricas para explicá-la. As teorias periféricas assumem que a causa da dor fantasma está nas terminações nervosas junto à lesão; já as teorias espinhais atribuem a causa da dor às alterações dentro da medula espinhal; enquanto as teorias centrais assumem que a dor fantasma é causada por um mecanismo dentro do encéfalo (Jensen \& Rasmussen, 1997; Nikolajsen \& Jensen, 2006).

Paralelamente a esse fenômeno da presença do membro fantasma, poderão vir associadas a sensação fantasma, a dor fantasma e a dor no coto, que se faz importante estabelecer uma distinção. A sensação fantasma pode ser considerada qualquer sensação, com exceção da dor, referente ao membro ausente. A dor no membro fantasma caracteriza-se como a sensação dolorosa referente ao membro ausente. E, por fim, a dor no coto seria caracterizada pela dor localizada apenas no próprio coto de amputação (Quadros, 2010). Ter claras essas definições não somente nos permite analisar a magnitude do problema como também o porquê de a conduta nessas variadas entidades clínicas serem inteiramente diferentes.

A intervenção, no que se refere à sensação fantasma, deve dar prioridade à validação do discurso e da sensação como real e não como resultado de uma reação imaginativa, alucinatória ou mesmo paranoica do paciente, possibilitando assim uma melhor reinserção social e reabilitação. Já em relação à dor no coto, é necessário um efetivo controle medicamentoso da dor, assim como avaliação e seguimento da intensidade e qualidade da dor referida pelo paciente, para que a intervenção seja o mais eficaz possível; considerando também a importância da avaliação multiprofissional que é necessária no controle da dor de forma global.

No que se refere aos tratamentos usados para controle da dor no membro fantasma, ainda não existe uma abordagem terapêutica padrão que seja eficaz, já que, como mencionado anteriormente, sua fisiopatologia não foi plenamente estabelecida. Assim, as abordagens terapêuticas de tratamentos 
podem ser basicamente divididas em três áreas: medicamentosa, de reabilitação (Fisioterapia, Psicologia, Terapia Ocupacional, entre outras) e cirúrgica, sendo que tais modalidades terapêuticas podem ser utilizadas de forma conjunta ou exclusiva.

Entendendo que a dor é um fenômeno complexo, subjetivo e único para cada pessoa, faz-se necessário compreender sua percepção idiossincrática, as estratégias de enfrentamento pessoais e os efeitos da dor sobre a qualidade de vida do paciente, assim como outros significados atribuídos a essa experiência. Dessa forma, as intervenções psicológicas, inseridas na abordagem terapêutica de reabilitação, poderão ser uma ferramenta eficaz no tratamento da dor.

Diante do exposto acima e devido ao caráter complexo que envolve o processo de adoecimento oncológico, bem como devido à necessidade de realização de tratamentos invasivos como a quimioterapia, a radioterapia e, ou, a cirurgia de amputação, faz-se necessário pensar a intervenção psicológica não apenas no âmbito de tratamento da dor fantasma, mas durante todo o processo que antecede a cirurgia. $\mathrm{O}$ atendimento psicológico poderá ajudar o paciente amputado e seus familiares a lidarem com essa nova situação, prevenindo maiores consequências emocionais, além de outros impactos como o próprio surgimento do fenômeno da dor no membro fantasma (Remor et al., 2003).

\section{INTERVENÇÃO CIRÚRGICA E OS ASPECTOS PSICOLÓGICOS}

Os pacientes com câncer e seus familiares necessitam constantemente de lidar com as várias fontes de estresse que estão presentes durante todo o processo de adoecimento: realizar exames para o diagnóstico e receber a confirmação diagnóstica, realizar os tratamentos, lidar com a volta à vida cotidiana e com a incerteza da recidiva, ou o encaminhamento para o tratamento nos cuidados paliativos (Cruzado, 2010). Entretanto, quando, além de todos esses fatores, o paciente necessita lidar com a possibilidade de realizar uma grande cirurgia como a de amputação, os impactos físicos e psicológicos tendem a ser potencializados.

Isto é, mesmo que a maioria dos pacientes amputados geralmente consiga se adaptar à nova situação e realizar uma reabilitação satisfatória, é frequente a comparência de períodos nos quais o paciente sofre profundas alterações emocionais. Em função disso, aproximadamente $50 \%$ dos pacientes amputados poderão necessitar de atendimento psicológico em alguma fase, desde a informação da realização da amputação até a reabilitação (Remor et al., 2003).

O psicólogo pode intervir nos momentos pré-operatório, transoperatório e pós-operatório, já que cada um desses momentos tem peculiaridades. Cada 
momento trará demandas distintas, porém, de forma geral, o objetivo do atendimento psicológico será minimizar a ansiedade e a angústia do paciente, possibilitando a expressão dos sentimentos e auxiliando na compreensão da situação vivenciada, proporcionando a verbalização de fantasias advindas do processo cirúrgico e também um clima de confiança entre o paciente e a equipe de saúde (Sebastiani \& Maia, 2005).

A intervenção psicológica tem a possibilidade de ocorrer de várias maneiras, sendo a psicoterapia sua base essencial. O termo psicoterapia abrange uma variedade de técnicas utilizadas para ajudar pessoas emocionalmente fragilizadas a modificarem seus comportamentos, pensamentos e moções, para que estas possam desenvolver melhores maneiras de lidar com situações vivenciadas de estresse, ou seja, desenvolver estratégias de enfrentamento adaptativas (Atkinson, Smith, Bem, Nolen-Hoeksema, \& Smith, 2002). Para melhor compreensão, o enfrentamento pode ser definido, segundo Nascimento, Castro, Amorim, e Bicudo (2011), como um mecanismo que o indivíduo desenvolve para enfrentar problemas ou situações estressantes.

Contudo é importante compreender que o atendimento psicológico se fundamenta na formação do vínculo terapêutico, o que, de modo genérico, é compreendido como a relação entre o psicoterapeuta e o paciente, bem como com os seus familiares, quando necessário (Remor et al., 2003). Porém é fundamental para o estabelecimento desse vínculo que o paciente aceite e queira receber o atendimento psicológico, caso contrário, o atendimento não colaborará com as necessidades terapêuticas.

Além da psicoterapia, a psicoeducação também pode estabelecer um papel importante na prevenção do desenvolvimento de alterações psicológicas ou psiquiátricas nos pacientes oncológicos e em seus familiares. A intervenção psicoeducativa é vista como uma importante ferramenta de intervenção no contexto oncológico, já que o câncer evoca um alto nível de ansiedade, de estresse e de incertezas nos pacientes e em seus familiares, devido ao estigma e ao desconhecimento sobre a doença e seus tratamentos. Nesse sentido, acredita-se que fornecer explicações corretas, em linguagem consonante ao nível cognitivo e à idade do paciente, possibilita um melhor entendimento do processo vivenciado, de forma mais realista, adequada e menos causadora de ansiedade e fantasias. Em relação ao procedimento cirúrgico e todas as consequências vinculadas a esse tratamento, fornecer as informações que o paciente queira em relação ao centro cirúrgico, hospitalização, e, no caso de amputação, a possibilidade do surgimento da sensação de membro fantasma pode prevenir o impacto psicológico vinculado às situações tão novas e desconhecidas pelos pacientes. Assim, a realização de intervenção psicoeducativa se faz necessária, uma vez 
que possibilita a compreensão aos pacientes acerca dos procedimentos a serem realizados (Alcântara, Shioga, Vieira Lima, Lage, \& Maia, 2013).

Contudo, mesmo com as intervenções psicoterapêutica e psicoeducativa, alguns pacientes ainda poderão manifestar a dor no membro fantasma. Por isso, para o tratamento específico desse fenômeno, existem diversas técnicas que podem ser usadas como recursos complementares à psicoterapia e à psicoeducação. Entre essas técnicas, destacam-se o biofeedback e a técnica do espelho proposta por Ramachandran (Mas Esquerdo, Fernández \& Sánchez, 2013).

As técnicas de biofeedback são utilizadas para modificar voluntariamente uma determinada resposta ou atividade fisiológica. Estudos apontam que a utilização de biofeedback em pacientes com dor no membro fantasma tem sido eficaz na redução e no controle da dor (Labrador, 2008; Harden et al., 2005). No Brasil, essa técnica ainda é pouco utilizada, e poucos estudos apresentam o uso da técnica com pacientes amputados.

A técnica do espelho também apresenta eficácia no tratamento da dor no membro fantasma, e é um recurso acessível e de fácil aplicação. Essa técnica consiste em colocar o membro sadio exatamente na mesma postura que ocuparia o membro que foi amputado. Quando o paciente observar sua imagem no espelho, o reflexo de seu membro sadio estará ocupando visualmente o lugar de localização do membro inexistente, de forma que se tem a ilusão de ótica que o "fantasma" foi regenerado. Além disso, durante o exercício, o paciente deve realizar movimentos com o membro sadio enquanto olha para o espelho, dessa forma, o paciente receberá a retroalimentação visual de que o membro fantasma é o que está se movimentando. Para que a técnica seja realmente efetiva, é necessário que os exercícios sejam realizados diariamente, com duração mínima entre cinco e dez minutos, por pelo menos três semanas. Desse modo se produz um fenômeno de sobreaprendizagem no qual a informação antiga e dolorosa é apagada gradualmente da memória e substituída por uma nova informação visual que indica que o membro está sadio (Mas Esquerdo et al., 2013).

No entanto, apesar dos avanços no tratamento da dor e das diversas formas de tratamento que podem ser utilizadas, a dor no membro fantasma ainda é considerada de difícil controle e manejo. Dessa forma, faz-se necessário perceber o caráter individual de cada sujeito e a sua relação com a dor, para que seja proposto o tratamento mais adequado à sua condição. Ademais, deve-se destacar a importância da informação e da psicoeducação na prevenção do surgimento da sensação fantasma e da dor no membro fantasma, principalmente pelo fato de tornar real, de forma antecipatória, algo que posteriormente poderá ser percebida como alucinação ou ilusão. Vale destacar novamente que todas as sensações 
relacionadas ao membro amputado são reais e bastante significativas na vida dos pacientes amputados.

A indicação da intervenção psicológica, portanto, deve ser pautada nas necessidades individuais de cada paciente. Esse trabalho se torna complexo ao englobar questões psíquicas, biológicas e sociais, necessitando da cooperação dos diversos profissionais envolvidos no processo cirúrgico e também no processo de adoecimento. Com isso, pode-se considerar a importância da atuação da Psicologia junto à equipe multidisciplinar para atuar com o paciente, com sua rede de apoio e com a equipe de saúde, visando a minimizar os sofrimentos decorrentes de todo esse processo, de forma a proporcionar o desenvolvimento da autonomia e corresponsabilização no processo de adoecimento de forma geral (Gabarra \& Crepaldi, 2009).

\section{DESCRIÇÃO DO CASO}

A paciente Maria (nome fictício que será utilizado para referir-se à paciente), 53 anos, dona de casa, recebeu o diagnóstico de sarcoma sinovial de tornozelo no início de 2014. Desde o diagnóstico até o mesmo período do ano seguinte, Maria realizou tratamento neoadjuvante de quimioterapia, seguido de cirurgia de amputação do membro inferior esquerdo devido à lesão e, posteriormente, toracotomia para retirada de metástases pulmonares.

Logo após o diagnóstico do tumor primário, antes de iniciar o tratamento oncológico de quimioterapia, foi iniciado o acompanhamento psicológico semanal, com o intuito de observar todo o processo de adoecimento e tratamentos realizados pela paciente. $\mathrm{O}$ primeiro atendimento foi solicitado pela irmã de Maria diretamente no Serviço de Psicologia do Hospital e, posteriormente, foram agendados e acordados com a paciente os atendimentos seguintes.

Ao longo de todo o tratamento, Maria sofreu várias perdas de ordens físicas, sociais e emocionais, decorrentes do processo de adoecimento, ou seja, da própria doença, dos tratamentos e seus efeitos secundários. Dessas perdas se destaca a realização da cirurgia de amputação.

Após a equipe de oncologia clínica optar por interromper a quimioterapia, devido a paciente apresentar grave toxicidade hematológica e não responder de forma esperada ao tratamento, Maria foi encaminhada à equipe de ortopedia para avaliação. Durante a consulta com a equipe da ortopedia, Maria foi informada da necessidade de realizar a cirurgia de amputação devido à agressividade do tumor. Após receber a notícia, Maria ficou em choque e bastante entristecida com a notícia, porém decidiu seguir com o tratamento oncológico e realizar o 
procedimento cirúrgico, o qual foi agendado para 30 dias após essa consulta.

No dia da cirurgia, observou-se que o maior medo apresentado pela paciente era o de não conseguir sobreviver. No pós-cirúrgico imediato, Maria relatava não imaginar como seria sua vida a partir daquele momento.

Logo nas primeiras semanas após a cirurgia, a paciente começou a relatar ter a sensação de que continuava com sua perna esquerda, ou seja, sensação fantasma, referindo sentir principalmente prurido. Por vezes, também foi observado um esquecimento em relação à amputação do membro, o que ocasionava tentativas de a paciente se levantar sozinha e colocar os dois pés no chão, contudo, ao sentar na cama, conseguia ter a percepção consciente de seu novo esquema corporal e não chegava a se levantar. Ou seja, a sensação fantasma existia, mas não era sentida como desconforto, provavelmente por ser conhecido e esperado por ela. Durante algumas noites, Maria apresentou dor fantasma, que diminuía com o uso de medicamentos, com massagens no membro contralateral e com o emprego da técnica do espelho. Apesar de apresentar bons recursos de enfrentamento para lidar com a perda do membro, Maria apresentava humor entristecido e sofrimento emocional, principalmente ao relacionar a perda do membro com a perda da função.

Ao se recuperar do pós-cirúrgico e começar um processo de reabilitação para colocar a prótese, Maria descobriu que estava com metástases pulmonares. Ao receber a notícia, a paciente ficou bastante fragilizada psiquicamente, chorosa e entristecida. Em seguida, foi proposta a realização da cirurgia para retirada dos nódulos. Então, em pouco mais de um mês após a notícia, Maria realizou a toracotomia, a qual apresentou uma difícil recuperação devido à modificação do esquema corporal e à dificuldade em deambular. Ao se recuperar do procedimento cirúrgico, Maria retornou o processo de reabilitação, com desejo da possível colocação da prótese.

Dois meses após a cirurgia no pulmão, Maria passou a apresentar sintomas de dor abdominal e no tórax, o que lhe causava bastante ansiedade. Após realizar exames para investigação diagnóstica, foi diagnosticada a existência de novas metástases pulmonares e também hepáticas. Naquele momento, ao serem propostas novas possibilidades terapêuticas para as metástases, apesar do medo e tristeza devido a todo processo vivenciado desde seu diagnóstico, Maria decidiu por não realizar nenhum tratamento e seguir com acompanhamento junto ao grupo de cuidados paliativos para controle de sintomas, em busca de uma melhora em sua qualidade de vida. Em seguida, devido à progressão da doença, a paciente foi a óbito. 


\section{INTERVENÇÃO PSICOLÓGICA}

As intervenções psicológicas realizadas com Maria ocorreram, aproximadamente, durante um ano e seis meses. $\mathrm{Na}$ entrevista inicial com a paciente, foram avaliados aspectos sociodemográficos, histórico psicológico e psiquiátrico, e aspectos relacionados ao diagnóstico e tratamentos a serem realizados. Assim, após o acolhimento e a escuta das demandas apresentadas por Maria, o objetivo terapêutico foi o de contribuir para a adaptação ao processo de adoecimento e tratamentos oncológicos.

Durante os primeiros atendimentos psicológicos, foram resgatados aspectos da história de vida, pensamentos, condutas e crenças em relação ao processo de adoecimento, ao tratamento de quimioterapia e aos eventuais períodos de hospitalização. Por meio disso, constatou-se que a paciente realizava tratamento para depressão, com acompanhamento psiquiátrico e uso de medicamentos havia cerca de dez anos antes do início do tratamento oncológico e que também apresentava episódios de fortes crises de ansiedade e insônia. As crises de ansiedade estavam sempre relacionadas a situaçôes gatilho, ou seja, conflitos familiares ou eventos de ordem pessoal.

De acordo com os relatos da paciente, ela tinha um filho dependente químico, que era a sua maior fonte de sofrimento emocional antes do início do tratamento oncológico. Por isso a relação de Maria com a situação de risco em que vivia seu filho e sua relação com o início do tratamento de quimioterapia foram o foco dos primeiros atendimentos. Desse modo, foram trabalhados pensamentos, crenças e o medo em relação à quimioterapia (alopecia, náuseas e vômito, astenia), e, de forma conjunta, foi abordada sua postura e percepção diante das questôes familiares.

Segundo Venâncio (2004), o psicólogo que trabalha na área da oncologia visa a manter o bem-estar psicológico do paciente, compreendendo e identificando os fatores emocionais que interferem em sua saúde. Além disso, a intervenção psicológica tem por objetivo prevenir e reduzir os sintomas emocionais advindos do câncer e da realização de seus tratamentos, levando o paciente a compreender o significado da experiência do adoecer, de forma a contribuir para as ressignificações desse processo.

Em um segundo momento, ao ser encaminhada para equipe de ortopedia, foi realizado acompanhamento junto a Maria e seu cônjuge no dia da consulta com a respectiva equipe. Durante a consulta, a paciente recebeu a informação de que realizaria a cirurgia de amputação do membro. Receber a notícia da amputação foi algo totalmente inesperado por Maria e seu familiar, já que, até aquele momento, sua grande fonte de sofrimento era a realização da quimioterapia. Por 
isso, devido ao impacto emocional da informação em relação ao procedimento cirúrgico proposto, foi realizado atendimento psicológico logo após consulta médica. Nesse momento, a escuta, o acolhimento e o suporte emocional foram fundamentais para ajudar a paciente a rever seus próprios recursos de enfrentamento para lidar com a situação. Posteriormente, devido à nova demanda apresentada (amputação), foram marcados atendimentos duas vezes por semana.

O mês de espera pela cirurgia de amputação foi chamado, nos atendimentos psicológicos, de período de preparação para a cirurgia. Nesse tempo, Maria apresentou uma série de questionamentos a respeito da adaptação ao novo corpo, de seu medo do procedimento cirúrgico, da possibilidade de realizar novamente quimioterapia, da perda da função do membro a ser amputado, de como conseguiria realizar suas atividades como dona de casa, além do medo de ficar dependente e necessitar de muitos cuidados. Dessa forma, o objetivo principal dos atendimentos psicológicos foi o de contribuir para o fortalecimento emocional diante da perda do membro e os aspectos físicos e funcionais relacionados a essa perda.

Durante os atendimentos, foram trabalhados pensamentos e crenças em relação ao procedimento cirúrgico, já que Maria referia medo e preocupação em relação à anestesia, ao procedimento, ao tempo de hospitalização e a outras questôes. Além disso, foi realizado um planejamento de alternativas para sua reabilitação e possíveis adaptações em casa para se adequar à nova situação, uma vez que, durante o início do processo de recuperação pós-cirúrgica, haveria a necessidade do uso de cadeiras de rodas e muletas. A realização desse planejamento teve como objetivo secundário fornecer à paciente maior segurança para enfrentar a situação futura, facilitar sua adaptação e fortalecer sua percepção de controle, já que os demais acontecimentos (doença e tratamentos) não poderiam ser controlados por ela. Consequentemente, o trabalho da relação com seu novo esquema e imagem corporal era fundamental para reduzir o impacto da mutilação provocada pela cirurgia. Foi abordado também o luto antecipatório em relação à perda do membro já que, diante de uma perda significativa, a vivência do luto seria fundamental no processo de enfrentamento e adaptação.

Além disso, nos atendimentos de preparação para a cirurgia, também foram trabalhadas as questôes relacionadas ao possível surgimento do membro fantasma, com a finalidade de prevenir o surgimento da dor no membro fantasma, que poderia instalar-se de forma crônica, devido aos riscos psíquicos apresentados pela paciente. Para auxiliar nessa abordagem, foram abordadas questôes relacionadas à fisiopatologia do membro fantasma, à sensação de dor fantasma e à dor no coto, pela psicoeducação, pois compreender e saber da possibilidade de acontecer tais fenômenos poderia reduzir a ansiedade e o medo da paciente, transformando o 
fenômeno em algo conhecido, deixando de ser novo e assustador. Também de forma preventiva, Maria foi treinada durante duas sessões para realizar a técnica do espelho, caso viesse a sofrer de dor no membro fantasma.

No dia da cirurgia, foi realizado acompanhamento de suporte à paciente no centro cirúrgico, desde sua entrada até o momento da anestesia, para minimizar o impacto emocional e reduzir a ansiedade no período pré-cirúrgico imediato, já que, segundo Remor et al. (2003), um aspecto fundamental do suporte emocional dentro da intervenção psicológica consiste no simples fato de que o terapeuta "esteja presente", como figura explícita de vínculo e como objeto seguro que o paciente pode localizar fisicamente em seu entorno. No pós-cirúrgico imediato, foram resgatadas questôes trabalhadas anteriormente durante os atendimentos psicológicos referentes ao momento posterior à amputação, para facilitar a adaptação ao momento vivenciado e melhorar o manejo da situação em domicílio.

Quinze dias após a amputação, Maria relatou ter a sensação fantasma, sentindo prurido e ardor no membro amputado, e também referiu episódios de dor fantasma durante algumas noites. Apesar de a paciente relatar a presença da sensação e da dor fantasma, eram episódios pontuais, pouco frequentes, e a presença da dor algumas vezes se relacionava a situações gatilho. Durante os atendimentos, a paciente relatava que outras questôes lhe causavam mais preocupação e sofrimento emocional, tais como a perda da função, principalmente por não conseguir realizar atividades domésticas, e a necessidade de depender dos familiares para realização de seus cuidados. Por esse motivo, foram trabalhadas questôes relacionadas à autonomia e ao manejo de cuidados em domicílio.

Para Remor et al. (2003), a angústia do paciente após a amputação não se limita à perda do membro em concreto, mas sim a todas as perdas resultantes da amputação, como a da autonomia funcional, em não poder realizar muitas atividades que antes desempenhava, e a grande mudança em sua imagem corporal. Em muitos casos, essas perdas podem ser vivenciadas como uma importante ruptura de suas possibilidades de ação, podendo acentuar sentimentos como inutilidade e dependência dos demais.

Ao longo de todo o processo, foram realizados atendimentos aos familiares de Maria (cônjuge, filha, nora e irmãs), entendendo que o paciente e a família são uma única unidade de cuidado e que a família geralmente se vê afetada quando um dos seus membros padece de uma doença que póe em risco a sua vida. Os objetivos da intervenção psicológica para os familiares, de forma geral, tendiam a contribuir para o fortalecimento de recursos de enfrentamento dos familiares e a realização de suporte emocional nos momentos necessários (Arranz, Barbero, 
Barreto, \& Bayés, 2003).

Durante consulta médica, Maria foi informada de sua metástase pulmonar e, após a comunicação da notícia, foi realizado atendimento de suporte emocional com a paciente e seus familiares (cônjuge e filha). Nos atendimentos seguintes, resgataram-se aspectos de seu modo de enfrentamento em relação ao processo de adoecimento, além de suas crenças e medos em relação às metástases pulmonares. $\mathrm{O}$ medo e as crenças apresentados em relação à situação vivenciada estavam muito relacionados com a lembrança da morte de seu pai, que havia falecido, havia poucos anos, de câncer no pulmão com metástases cerebrais. Por isso foram então trabalhadas questôes relacionadas ao processo de adoecimento de seu pai e ao seu próprio processo.

Entretanto, devido à progressão da doença, Maria seria submetida a outro procedimento cirúrgico para a retirada das metástases pulmonares. Por esse motivo, foram trabalhadas questôes que previamente haviam sido foco da intervenção psicológica: o procedimento cirúrgico, sua reabilitação e sua dependência de cuidadores.

Por conta da cirurgia, a paciente precisou interromper seu processo de reabilitação para colocar a prótese que substituiria o membro amputado anteriormente. Saber que continuaria dependendo de terceiros para a realização de cuidados e tarefas, e a angústia de não saber se a cirurgia controlaria a evolução da doença lhe causavam tristeza, ansiedade e a sensação de fracasso. Por isso, o objetivo das intervenções passou a ser o resgate da autonomia e da sensação de controle de sua própria vida, apesar da limitação física, e a redução da angústia em relação a acontecimentos futuros.

Segundo Melo, Valero, e Menezes (2013), um recurso que pode ser utilizado nesse contexto, durante as intervenções psicológicas, é a exploração das fantasias geradas diante dos medos e perdas já vividos pelo paciente, além de buscar favorecer a elaboração desses conteúdos, das expectativas e frustrações, propiciando novas possibilidades de adaptação à situação vivenciada.

Após a retirada das metástases pulmonares, a paciente seguia com o objetivo de conseguir colocar a prótese e voltar a realizar suas atividades diárias, porém esse processo foi novamente interrompido ao ser constatada a presença de novas metástases, dessa vez pulmonares e hepáticas. Diante desse novo quadro diagnóstico, Maria apresentou grande fragilidade emocional, relatando sentirse bastante entristecida, desanimada e com medo da morte e do sofrimento. A partir daquele momento, o objetivo terapêutico passou a ser o de contribuir com o fortalecimento de novos recursos de enfrentamento, bem como dos já utilizados para lidar com o diagnóstico de múltiplas metástases. Além disso, 
buscou-se fortalecer a autonomia da paciente em relação à tomada de decisão na escolha do tratamento e na melhora da qualidade de vida. Maria decidiu, então, seguir com acompanhamento exclusivo com a equipe de cuidados paliativos. Posteriormente, a paciente veio a falecer na instituição hospitalar, acompanhada de seus familiares.

\section{RESULTADOS OBTIDOS}

A partir das intervenções psicológicas, foram alcançados vários dos objetivos que foram determinados de acordo com a escuta da paciente e as demandas apresentadas. Inicialmente, ao se deparar com o diagnóstico e o tratamento quimioterápico, Maria encontrou uma ressignificação para sua vida. Passou a lidar com os problemas de ordem familiar, relacionados a seu filho, por uma outra perspectiva, ao vivenciar uma situação extrema, na qual sua vida estava em risco. Assim, durante a realização da quimioterapia, Maria diminuiu os episódios de crises de ansiedade, mesmo quando surgia alguma situação gatilho, e conseguiu lidar com o tratamento utilizando recursos de enfrentamento adaptativos, apesar de permanecerem os aspectos emocionais relativos à depressão.

No momento de preparação para cirurgia de amputação, a partir da psicoterapia e da psicoeducação, foram realizados planejamentos para que a paciente conseguisse reorganizar sua casa para melhorar sua mobilidade e favorecer sua independência. Apesar da perda da função, que acarretou na dependência dos familiares para realizar atividades, Maria conseguia realizar algumas tarefas devido às adaptaçôes, como tomar banho (devido à barra de apoio), ter um melhor acesso ao quarto (transferiu seu quarto para parte inferior da casa), além de ter um melhor acesso ao banheiro e à cozinha. Todas essas mudanças ocorreram antes mesmo da realização da cirurgia, o que facilitou no processo de adaptação, principalmente nos períodos em que Maria estava andando com a ajuda de muletas.

Como era conhecida a data em que ocorreria a amputação, Maria planejou um momento onde poderia se despedir de sua perna. No final de semana que antecedeu a cirurgia, Maria, juntamente com os familiares, foi para a praia e, de fato, pôde vivenciar o luto antecipatório relacionado à perda desse membro.

A psicoeducação e o treinamento na técnica do espelho antes da realização da cirurgia foram fundamentais para que a paciente não apresentasse, de forma significativa, a dor fantasma e, quando a apresentasse, conseguisse um bom controle, empregando a técnica aprendida e os medicamentos. A paciente relatava que "Se não soubesse do fenômeno antes de acontecer, acharia que estava louca”. Assim, a prevenção foi fundamental e eficaz para que a dor no membro 
fantasma não se tornasse algo que incrementaria todo o sofrimento emocional já vivenciado pela paciente nem se tornasse um evento crônico, como visto em vários casos na literatura.

Em concreto, a intervenção psicológica poderá ajudar o paciente a enfrentar, de maneira realista, a doença, a intervenção cirúrgica, as sequelas advindas da amputação, a dor, o membro fantasma e os problemas que forem surgindo ao longo do processo (Remor et al., 2003). Assim, os resultados obtidos estão consonantes com o que a literatura apresenta como benefício e objetivo das intervençōes psicológicas.

Respeitar a autonomia da paciente e facilitar recursos para tomada de decisão favoreceu a paciente na realização daquilo que desejava, dentro dos limites que a doença lhe colocou. Em um primeiro momento, a paciente relatou querer realizar a cirurgia para a retirada da metástase pulmonar, entretanto, quando surgiram as múltiplas metástases, apesar de sua família querer que a paciente seguisse com outros tratamentos, ela teve a possibilidade de escolher parar com os possíveis tratamentos (cirurgia e quimioterapia) e seguir com o controle dos sintomas.

Apesar do histórico familiar e psiquiátrico da paciente, as intervenções psicológicas alcançaram seus objetivos, sendo o primeiro deles respeitar a autonomia e o tempo da paciente. Cada objetivo foi construído de forma conjunta com Maria e de acordo com cada momento vivenciado. Entre tantos eventos que Maria não podia controlar, era nas intervenções psicológicas que ela podia manifestar seus medos, seu choro contido, suas angústias e preocupações. Abordar temas de perdas, de morte e de sofrimento somou a essa realidade pequenas conquistas, sonhos, desejos e alegrias.

\section{CONSIDERAÇÕES FINAIS}

Podemos considerar que um paciente, ao enfrentar uma doença estigmatizada como o câncer, está suscetível a sofrer danos físicos e emocionais que muitas vezes são irreparáveis. No caso de Maria, o medo vinculado à crença de ter uma doença oncológica agressiva e devastadora foi algo que se concretizou, pois, apesar dos avanços no tratamento das doenças oncológicas, ainda é constante o sofrimento dos pacientes devido às consequências desses tratamentos que, como relatado no caso, algumas vezes são mutiladores e limitantes.

Compreender o ser humano, sua complexidade e singularidade ainda é um desafio para os profissionais da saúde. No relato do caso apresentado, a paciente conseguiu se adaptar às sucessivas perdas, superar o período de dor no membro 
fantasma e fortalecer sua autonomia diante de seu processo de adoecimento. Porém a tristeza e o sofrimento fizeram parte de todo o processo, e essa reação emocional foi adaptativa e esperada diante de toda a situação vivenciada.

Pelas experiências relatadas, portanto, percebe-se a importância que existe em os profissionais da saúde estarem preocupados, atentos e preparados para as diversas perdas que acometem os pacientes, já que cada perda pode estar vinculada à possibilidade, ao desejo e à esperança de cura. Poder acolher e respeitar o sofrimento do paciente, quando cada tentativa de cura não acontecer, deveria ser um papel a ser desempenhado por toda a equipe, não somente pelo profissional psicólogo, pois se deve entender que o cuidar do outro se faz necessário em todos os momentos do processo de adoecimento e que, para cuidar, é fundamental considerar o paciente como um ser único, que tem seu próprio conceito de qualidade de vida. 


\section{REFERENNCIAS}

Alcântara, T. V. D., Shioga, J. E. M., Vieira Lima, M. J., Lage, A. M. V., \& Maia, A. H. N. (2013). Intervenções psicológicas na sala de espera: estratégias no contexto da Oncologia Pediátrica. Revista da SBPH, 16(2), 103-119.

Arranz, P., Barbero, J., Barreto, P., \& Bayés, R. (2003). Intervención emocional en cuidados paliativos. Barcelona: Ariel.

Atkinson, R. L., Atkinson, R. C., Smith, E. E., Bem, D. J., Nolen-Hoeksema, S. \& Smith, C. D. (2002). Introdução à Psicologia de Hilgard. (13a ed.). Porto Alegre: Artmed.

Corrêa, D. S., Cunha, I. W. D., Carvalho, A. L., Soares, F. A., Fauzoni, R., \& Lopes, A. (2005). Sarcoma sinovial de extremidades com doença localizada ao diagnóstico: tratamento e padrões de recidiva em 57 casos. Revista do Colégio Brasileiro de Cirurgióes, 32(6), 304-309.

Coutinho, M. \& Moura, R. (2004). Aspectos psicológicos relacionados à reabilitacão do paciente com amputação. In A. Pedrinelli. Tratamento do paciente com amputação. São Paulo: Roca.

Cruzado, J. A. (2010). Tratamiento psicológico en pacientes con cáncer. Madrid: Síntesis.

Demidoff, A. D. O., Pacheco, F. G., \& Sholl-Franco, A. (2007). Membrofantasma: o que os olhos não veem, o cérebro sente. Ciências \& Cognição, 12, 234-239.

Faria, S. S. \& Silva, P. L. (2014). Revisão sistemática sobre tratamento medicamentoso para dor no membro fantasma. Revista Neurociência, 22(2), 177-188.

Flor, H., Birbaumer, N., \& Sherman, R. A. (2001). Dolor de miembro fantasma. Revista Sociedad Española de Dolor, 8, 327-331.

Gabarra, L. M. \& Crepaldi, M. A. (2009). Aspectos psicológicos da cirurgia de amputação. Aletheia, 30, 59-72.

Harden, R. N., Houle, T. T., Green, S., Remble, T. A., Weinland, S. R., Colio, S., ... Kuiken, T. (2005). Biofeedback in the treatment of phantom limb pain: a time-series analysis. Applied psychophysiology and biofeedback, 30(1), 83-93. 
Instituto Nacional do Câncer. (2016). O que é câncer?. Recuperado a partir de http://www1.inca.gov.br/conteudo_view.asp?id=322

Jensen, T. S. \& Rasmussen, P. (1997). Phantom pain and other phenomena after amputation. In P. D. Wall \& R. Melzack (Eds), Textbook of Pain. (3a ed., pp. 861-865). Nova Iorque: Churchill Livingstone.

Labrador, F. J. (2008). Técnicas de modificación de conducta. Madrid: Pirámide.

Manoel, W. J., Sarmento, B. J. D. Q., Silveira Júnior, L. D. P., Abreu, D. C. B. D., Abreu Neto, I. P., \& Ferreira, E. C. (2008). Sarcomas de partes moles: resultados do tratamento dos tumores de baixo grau. Revista Brasileira de Cancerologia, 54 (1), 17-24.

Mas Esquerdo, J., Fernández, R. M., \& Sánchez, J. I. R. (2013). Tratamiento neuropsicológico de "dolor de miembro fantasma": a propósito de un caso. Sanidad Militar, 69(3), 195-202.

Melo, A. C. D., Valero, F. F., \& Menezes, M. (2013). A intervenção psicológica em cuidados paliativos. Psicologia, Saúde \& Doenças, 14(3), 452-469.

Nascimento, A. N., Castro, D. S., Amorim, M. H. C., \& Bicudo, S. D. S. (2011). Estratégias de enfrentamento de familiares de mulheres acometidas por câncer de mama. Ciência, Cuidado e Saúde, 10(4), 789-794.

Nikolajsen L. \& Jensen T. S. (2006). Phantom limb. In S. McMahon, M. Koltzenburg (Eds.), Wall \& melzack's textbook of pain (5a ed., pp. 961-972). Londres: Elsevier.

Pellizzon, A. C. A., Salvajoli, J. V., \& Novaes, P. E. R. S. (2002). Cirurgia conservadora, radioterapia externa e reforço de dose com braquiterapia de alta taxa de dose: uma nova perspectiva no tratamento de sarcomas de partes moles do adulto. Radiologia Brasileira, 35(2), 89-92.

Pires, M. C. B. \& Bastos, S. M. (2013). Terapia Ocupacional: contribuiçôes e perspectivas no atendimento à pessoa amputada. São Paulo: Livre Expressão.

Quadros, L. D. F. D. C. (2010). A prevalência e a repercussão psicológica e funcional da dor e sensação fantasma na amputação do membro inferior por isquémia avançada. (Tese de Doutorado). Universidade de Lisboa, Faculdade de Medicina de Lisboa, Lisboa, Portugal.

Remor, E., Ulla, S., \& Arranz, P. (2003). El psicólogo en el ámbito hospitalario. 
Bilbao: Desclee De Brouwer.

Santos, C. E. R., Rezende, J. F. N., Carvalho, G. S. S., \& Nunes, F. N. (2015). Sarcomas de partes moles. Rio de Janeiro: Cirurgia Online. Recuperado a partir de http://www.cirurgiaonline.com.br/site/images/stories/pdf/sarcomas\%20 de $\% 20$ partes $\% 20$ moles $\% 20$ em $\% 20$ dermatologia $\% 20$ (trocar $\% 20$ por $\% 20$ este\%20atualizado).pdf

Schuler, T. A., Zaider, T. I., \& Kissane, D. W. (2012). Family grief therapy: a vital model in oncology, palliative care and bereavement. Family Matters, 90, 77.

Sebastiani, R. W. \& Maia, E. M. C. (2005). Contribuições da Psicologia da Saúde-Hospitalar na atenção ao paciente cirúrgico. Acta Cirúrgica Brasileira, 20(Suppl. 1), 50-55.

Venâncio, J. L. (2004). Importância da atuação do psicólogo no tratamento de mulheres com câncer de mama. Revista Brasileira de Cancerologia, 50(1), 5563. 